\title{
Modeling the Initial Flame Acceleration in an Obstructed Channel using Large Eddy Simulation
}

\author{
C.Johansen ${ }^{* * 1}$ and G. Ciccarelli*†2 \\ ${ }^{1}$ Department of Mechanical and Manufacturing Engineering, University of Calgary, \\ Calgary, Canada \\ ${ }^{2}$ Department of Mechanical and Materials Engineering, Queen's University, Kingston, \\ Canada
}

July 1, 2013

\begin{abstract}
The propagation and acceleration of a flame surface past obstructions in a closed square channel was investigated using large eddy simulation. The dynamic Smagorinsky-Lilly subgrid model and the Boger flame surface density combustion model were used. The geometry is essentially two-dimensional with fence-type obstacles distributed on the top and bottom surfaces, equally spaced along the channel length at the channel height. Flame propagation, however, is three dimensional as ignition occurs at a point at the center of the channel cross-section. The effect of obstacle blockage ratio on the development of the flame structure was investigated by varying the obstacle height. Three-dimensional cases were simulated from the initiation of a combustion kernel through spark ignition to the acceleration of the flame front at speeds up to $80 \mathrm{~m} / \mathrm{s}$. The transition from laminar flame propagation to turbulent flame propagation within the "thin reaction zone" regime was observed in the simulations. By analyzing the development of the three dimensional flame surface and unburned gas flow field, the formation of several flame structures observed experimentally are explained. Global quantities such as the total flame area and centerline flame velocity were ascertained and compared to the experimental data. High amplitude oscillations in the centerline flame velocity were found to occur from a combination of the unburned gas flow field and fluctuations in the volumetric burning rate.
\end{abstract}

\section{Nomenclature}

\section{Abbreviations}

CFD $\quad$ computational fluid dynamics

DDT deflagration-to-detonation transition

LES $\quad$ large eddy simulation

$\mathrm{OH} \quad$ hydroxyl radical

PDE $\quad$ pulse detonation engine

PIV $\quad$ particle image velocimetry

PLIF $\quad$ planar laser induced fluorescence

SIMPLE $\quad$ semi-implicit method for pressure-linked equations

UDF user defined function

\footnotetext{
${ }^{* *}$ Corresponding author. Email: johansen@ucalgary.ca

†*Co-author. Email: ciccarel@me.queensu.ca
} 


\section{English}

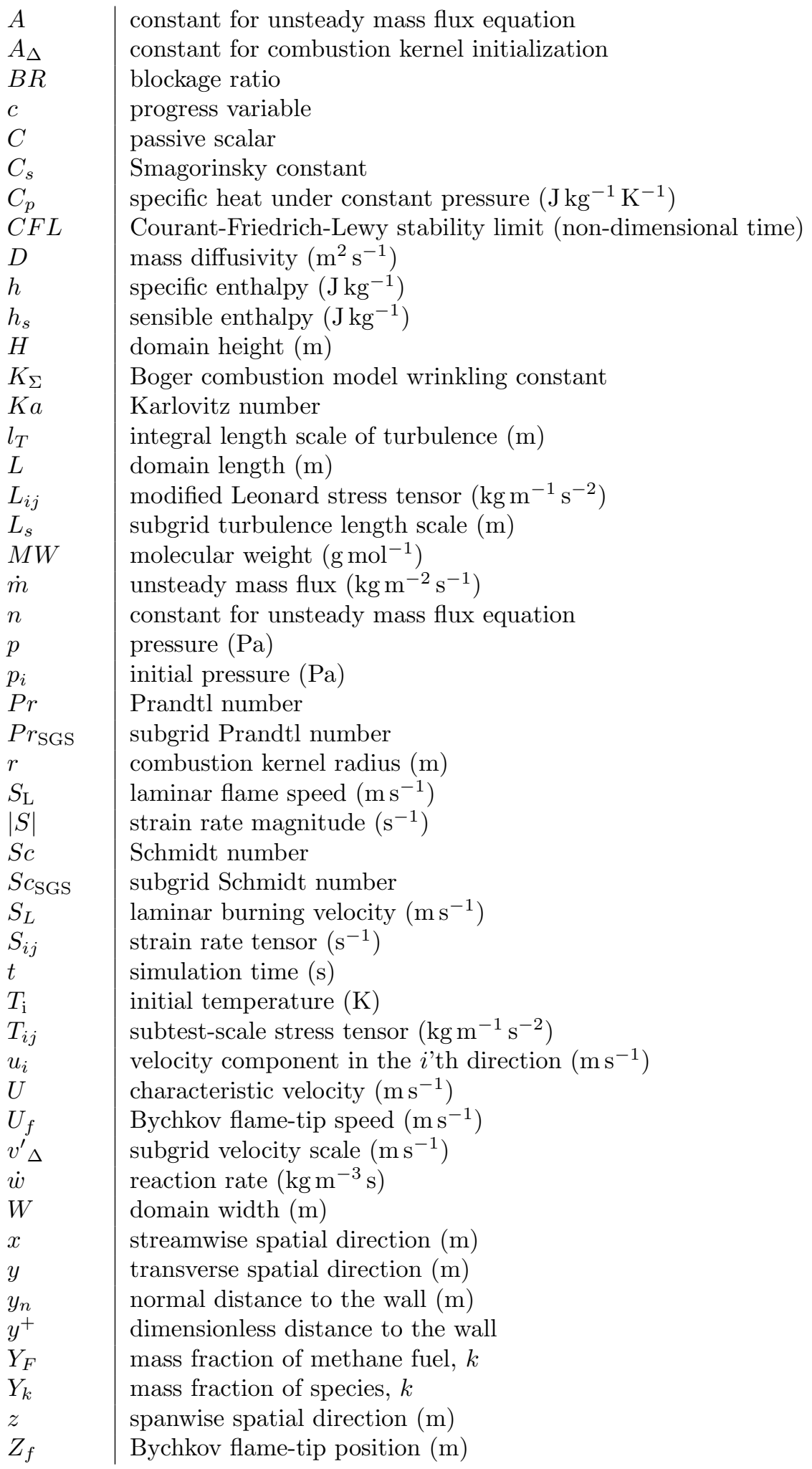




\section{Greek}

\begin{tabular}{l|l}
$\delta_{\Delta}$ & initial numerical flame thickness $(\mathrm{m})$ \\
$\delta_{L}$ & laminar flame thickness $(\mathrm{m})$ \\
$\delta_{R}$ & reaction zone thickness $(\mathrm{m})$ \\
$\Delta$ & grid node spacing $(\mathrm{m})$ \\
$\Delta_{K}$ & Boger combustion model filter width $(\mathrm{m})$ \\
$\Delta H_{\text {comb }}$ & Boger combustion model filter width $(\mathrm{m})$ \\
$\Delta_{t}$ & time step size $(\mathrm{s})$ \\
$\eta$ & Kolmogorov length scale $(\mathrm{m})$ \\
$\tilde{\vartheta}$ & favre-averaged variable \\
$\bar{\vartheta}$ & filtered variable \\
$\rho$ & density (kg $\left.\mathrm{m}^{-3}\right)$ \\
$\rho_{u}$ & density of unburned gas $\left(\mathrm{kg} \mathrm{m}^{-3}\right)$ \\
$\kappa$ & von Kármán constant $(=0.41)$ \\
$\lambda$ & thermal conductivity $\left(\mathrm{W} \mathrm{m}^{-1} \mathrm{~K}^{-1}\right)$ \\
$\mu$ & dynamic viscosity $\left(\mathrm{kg} \mathrm{m}^{-1} \mathrm{~s}^{-1}\right)$ \\
$\mu_{\mathrm{SGS}}$ & subgrid viscosity $\left(\mathrm{kg} \mathrm{m}^{-1} \mathrm{~s}^{-1}\right)$ \\
$\sigma$ & expansion ratio \\
$\sigma_{i j}$ & viscous stress tensor $\left(\mathrm{kg} \mathrm{m}^{-1} \mathrm{~s}^{-2}\right)$ \\
$\Sigma$ & flame surface density $\left(\mathrm{m}^{2} \mathrm{~m}^{-3}\right)$ \\
$\tau_{\mathrm{c}}$ & chemical time scale $(\mathrm{s})$ \\
$\tau_{\eta}$ & Kolmogorov time scale $(\mathrm{s})$ \\
$\tau_{i j}$ & subgrid stress tensor $\left(\mathrm{kg} \mathrm{m}^{-1} \mathrm{~s}^{-2}\right)$ \\
$\tau_{\mathrm{w}}$ & wall shear stress $\left(\mathrm{kg} \mathrm{m}^{-1} \mathrm{~s}^{-2}\right)$ \\
$\omega$ & vorticity ( $\left.\mathrm{s}^{-1}\right)$
\end{tabular}

\section{Subscripts}

\begin{tabular}{l|l}
$\mathrm{SGS}$ & subgrid scale \\
$\mathrm{c}$ & chemical scale \\
$\eta$ & Kolmogorov scale \\
$i, j, k$ & index counts in the three grid directions; $x, y, z$ components of a quantity \\
$\mathrm{K}$ & Boger combustion model \\
$\mathrm{u}$ & unburned gas
\end{tabular}

\section{Introduction}

The study of flame acceleration in an obstructed channel has applications ranging from explosion safety to the development of pulse detonation engines (PDEs) [1,2]. Under certain conditions, flame acceleration can lead to deflagration-to-detonation transition (DDT). A detonation wave is a supersonic mode of combustion which produces a large dynamic pressure. From the onset of spark ignition at the closed end of a channel, the development of the unburned gas flow field has a large influence on the rate of flame acceleration prior to DDT [3]. The unburned gas flow field is driven by the expansion of combustion products behind the flame front and becomes distorted due to the geometry of the confinement upstream. In the present study, obstacles mounted on the top and bottom channel surfaces are responsible for distorting the unburned gas flow field. The generation of a shear layer from the obstacle tips results in the production of turbulence, which increases the transport of mass, momentum, and energy in the flow. Large-scale unburned gas flow distortions around the obstacles cause the flame to "fold" thereby increasing the flame area. As the flame propagates into regions characterized by high levels of turbulence, small-scale wrinkling further increases the total flame area. An increase in the volumetric burning rate resulting from flame area production causes the expansion rate of the combustion products to increase thereby increasing the unburned gas velocity. A feed-back loop is formed between the volumetric burning rate and the unburned gas velocity, which leads 
to flame acceleration. In the presence of obstacles, large amplitude fluctuations of the flame-tip velocity are observed experimentally [4]. These oscillations are due to the acceleration and deceleration of the unburned gas as it flows through the openings between obstacles. The amplitude of the oscillations are more significant in tests with the higher blockage ratio $(B R)$ obstacles because of the more severe flow contraction. At the later stages of flame acceleration prior to DDT, shock-flame interactions become the dominant mechanism responsible for flame acceleration $[1,5]$.

In this study, attention is focused on the initial stages of flame acceleration where shock-flame interactions and other compressibility effects are negligible. Understanding the early stages is essential as they are responsible for creating the conditions required for DDT. Because the initial-to-final time scale ratio associated with DDT is large, the majority of time required to achieve DDT is consumed early on in the initial stages of flame acceleration. In the context of PDEs, which must operate at high frequencies to provide adequate thrust, effort spent minimizing the time duration of the initial stages can greatly improve the performance of the engine [6]. In the context of explosion safety, inhibiting the rate of flame area production during the initial stages can reduce the total overpressure and perhaps prevent a detonation initiation event [1].

Recent numerical simulations of flame propagation in obstructed channels have given insight into the mechanisms responsible for flame acceleration. Gamezo et al. [7] simulated flame acceleration in an obstructed channel from spark ignition up to the initiation and propagation of a detonation wave. Simulations were completed on a two-dimensional, adaptive grid, which allowed the hydrogen-air flame thickness to be resolved with approximately 18 nodes. The simulation used single step chemistry modeled with Arrhenius kinetics and the grid was refined dynamically at each time step based on gradients of density, pressure, velocity, and mixture composition. Although the flame thickness was resolved, the turbulence field was spatially underresolved and no turbulence model was used. Bychkov et al. [8] performed simulations of the initial stages of flame acceleration in an obstructed channel prior to DDT for the purpose of developing an analytical model to predict changes in the flame speed. Similarly, the turbulence field was under-resolved and no turbulence model was used.

A numerical study, which was entirely focused on predicting the development of the unburned gas flow field ahead of the flame and included the effects of turbulence, was performed by Johansen and Ciccarelli [9]. An LES of the transport of a passive scalar ahead of the flame front was performed and compared to experiments performed by Johansen and Ciccarelli [4]. These experiments involved using schlieren photography to visualize stoichiometric methane-air explosions at an initial pressure and temperature of $p_{i}=47 \mathrm{kPa}$ and $T_{i}=293 \mathrm{~K}$, respectively. The channel had a total length of $2.44 \mathrm{~m}$ and a square cross-section of $7.6 \mathrm{~cm} \times$ $7.6 \mathrm{~cm}$. Obstacles of varying blockage ratios $(B R=0.33,0.5,0.67)$ were distributed along the channel top and bottom surfaces spaced at a distance equivalent to the channel height. A novel visualization technique was developed where a small amount of helium tracer gas was injected ahead of the flame front. Figure 1 illustrates how the visualization technique was used in a test with $0.5 B R$ obstacles. Helium tracer gas was injected downstream of the ignition point transversely via a port through the top surface of the channel between obstacles prior to ignition. Using schlieren photography the displacement of the helium/mixture interface from the expanding combustion products was observed. Observation of both the simulation results and experimental data revealed the unsteady formation of laminar vortices behind the obstacles' trailing edges and a subsequent transition to turbulence in the shear layer. After validating the numerical models with this experimental data, further information such as the evolution of velocity, vorticity, and pressure distributions were extracted from the simulations. It was found that three-dimensional effects were important to the early development of the core flow and of the shear layers. Figure 2 shows simulation predictions of the three-dimensional evolution of the helium/gas interface at several stages of flow acceleration. Contours of spanwise vorticity $\left(\omega_{z}\right)$ are shown on the side wall to illustrate the shedding of vortices from the obstacle tips. Stream traces are overlaid on the image and show the contraction and expansion of the flow near the obstacles. Contours of subgrid viscosity ratio are displayed on an iso-surface of the passive scalar and indicate high levels of turbulence production near the leading edge of the obstacle tips. It was found that the obstacle $B R$ plays an important role in determining the flow structure in the unburned gas.

Recently, simulations of flame acceleration past obstructions have been performed [10-14] in relatively short-length channels. Flame surface density combustion models and LES were used to treat the effect of chemical reactions and turbulence on the mean flow field. The mixture simulated was stoichiometric methane-air. In one of the studies, three-dimensional obstacles were distributed along the channel centerline [10]. During flame propagation, the exit of the channel was open to the atmosphere, allowing combustion 

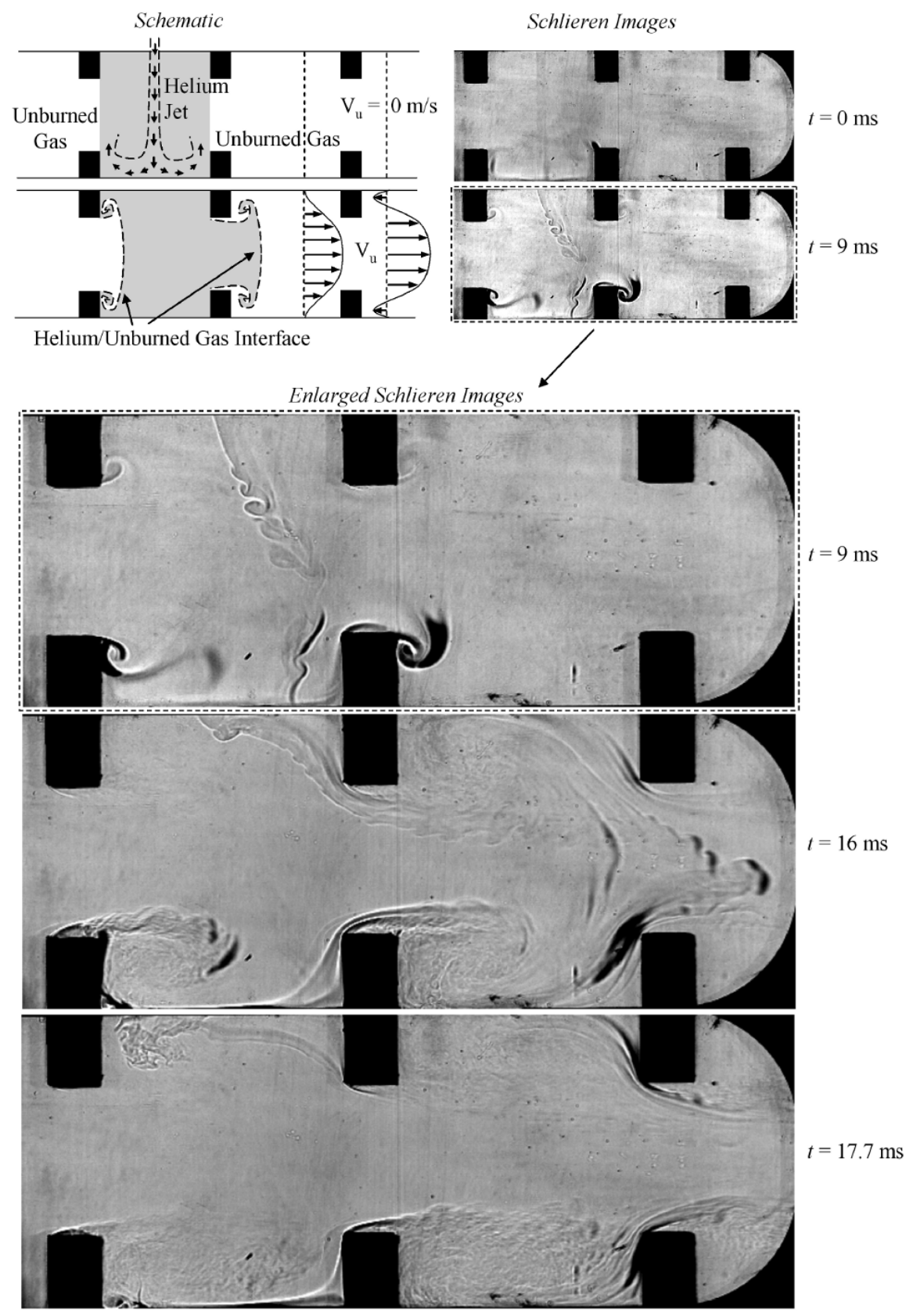

Figure 1: Schlieren photographs and schematic of the development of the unburned gas flow field ahead of the flame using helium gas as a tracer. Adapted from [4]. 

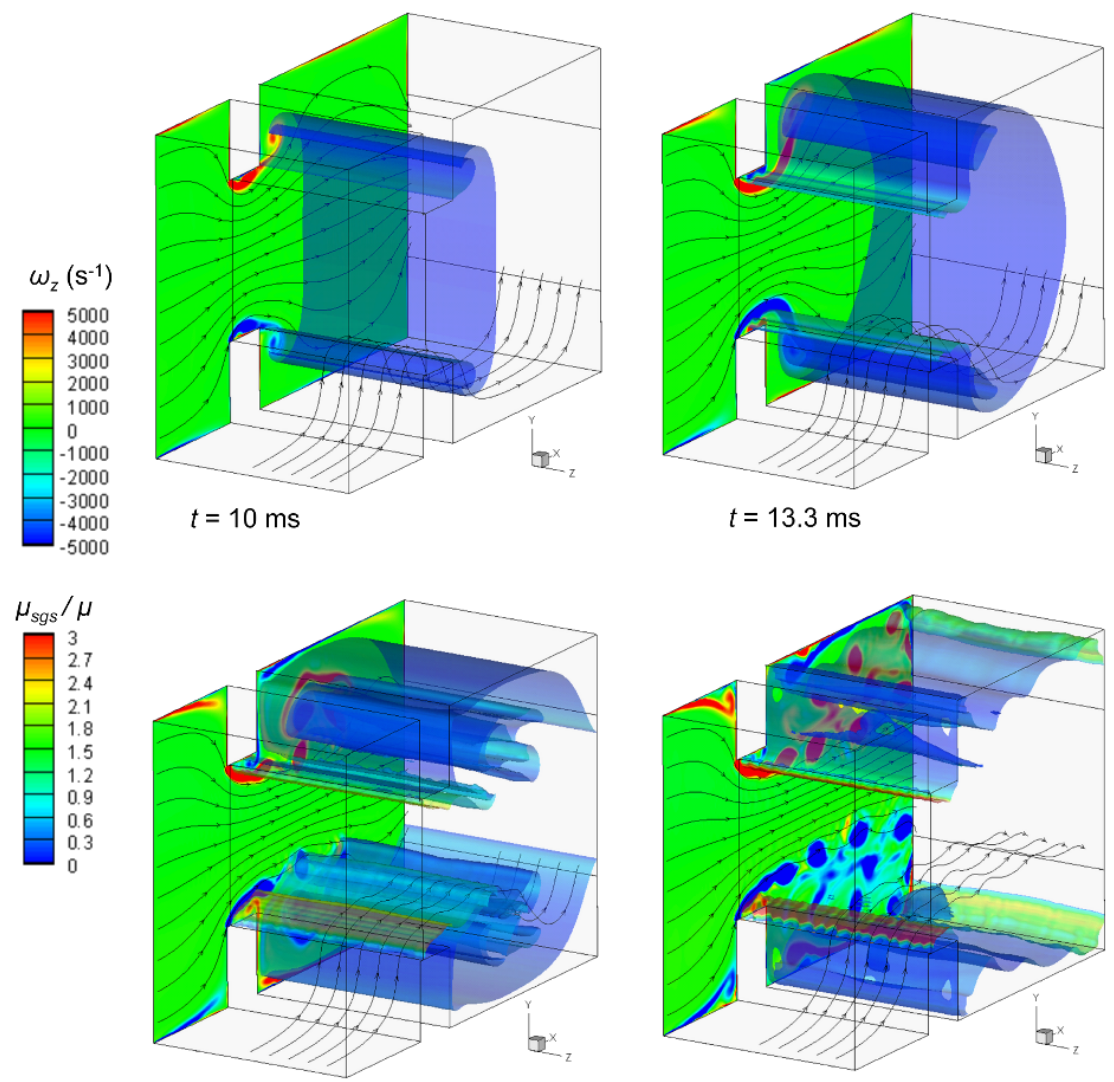

$t=16.7 \mathrm{~ms}$

$t=20 \mathrm{~ms}$

Figure 2: 3D flow field development $(B R=0.5, W / H=0.67, L / H=1.41)$. Standard Smagorinsky model used. Distribution of subgrid viscosity ratio is shown as contours on $C=0.5$ iso-surface. Distribution of $z$ vorticity is shown on $x-y$ plane. Stream-traces are distributed along $y$ axis at the $-z$ extent of domain and near bottom wall surface. Reproduced from [9]. This image should be published in color. 
products to escape. Favorable agreement between experimental data [15] and simulation predictions was achieved. Flow visualization and the velocity field were obtained from high-speed laser sheet flow visualization images and particle image velocimetry (PIV). There was also favorable agreement between the experiment and simulation predictions of the flame-tip speed and wall pressure distribution along the length of the channel. In another work, PIV and OH planar laser induced fluorescence (PLIF) measurements were obtained and compared to simulations of flame propagation past three-dimensional surface mounted obstacles [11]. Similarly, a flame surface density combustion model and LES were used to close the system of equations. There was good agreement between simulation predictions and experimental measurements of the surface pressure and flame structure. It was found that the combustion model was sensitive to the choice of filter width, which affects the resolution of the reaction zone.

In this work, LES and a simple flame surface density combustion model are used to simulate flame acceleration through a series of two-dimensional obstacles along a relatively long channel length. The threedimensional simulations capture the transition to turbulence at various obstacle $B R$ configurations. With the relatively long computational domain, high flame velocities are achieved. Effective three-dimensional comparisons are made between experimental schlieren images and simulation predictions. In addition, the flame area and flame velocity are extracted from both the simulation and experiment to provide for quantitative comparisons. Finally, the computational fluid dynamics (CFD) model is used as an investigative tool to help explain the formation of flame structures that are observed experimentally.

The main contribution of this work is the detailed analysis of the three-dimensional unburned gas flow field development and its effect on the flame shape for various obstacle $B R$. The previously described simulations mainly focus on the flame-tip speed and pressure development in channels with one to a few center-mounted obstacles [10-14]. Although they discuss the three-dimensional flow field, they do not explain how the unburned gas is affected by obstacle $B R$ and how changes in the unburned gas development affect the flame shape. Moreover, many of the studies are restricted to a single obstacle $B R$ configuration. The higher flame speed simulations performed by Gamezo et al. focus on the DDT phenomena and are mainly two-dimensional [7]. Therefore, this work focuses on the effect of fence-type obstacle $B R$ on the three dimensional flame shape development that occurs at relatively high flame-tip speeds but before the onset of compressibility effects in the unburned gas flow.

\section{Simulation Setup}

A low-Mach number formulation of the compressible filtered governing equations was solved:

$$
\begin{gathered}
\frac{\partial \bar{\rho}}{\partial t}+\frac{\partial \bar{\rho} \tilde{u}_{i}}{\partial x_{i}}=0 \\
\frac{\partial \bar{\rho} \tilde{u}_{i}}{\partial t}+\frac{\partial \bar{\rho} \tilde{u}_{i} \tilde{u}_{j}}{\partial x_{i}}+\frac{\partial \bar{p}}{\partial x_{i}}-\frac{\partial \tilde{\sigma}_{i j}}{\partial x_{j}}=-\frac{\partial}{\partial x_{j}} \underbrace{\left[\bar{\rho}\left(\widetilde{u_{i} u_{j}}-\tilde{u}_{i} \tilde{u}_{j}\right)\right.}_{\text {subgrid stress }}] \\
\frac{\partial \bar{\rho} \tilde{h}_{s}}{\partial t}+\frac{\partial \bar{\rho} \tilde{u}_{i} \tilde{h}_{s}}{\partial x_{i}}-\frac{\partial}{\partial x_{i}}\left(\lambda \frac{\partial \tilde{T}}{\partial x_{i}}\right)=\underbrace{\overline{\dot{w}_{T}}}_{\begin{array}{c}
\text { filtered } \\
\text { heat release }
\end{array}}-\frac{\partial}{\partial x_{j}} \underbrace{\left[\bar{\rho}\left(\widetilde{u_{i} h_{s}}-\tilde{u}_{i} \tilde{h}_{s}\right)\right.}_{\begin{array}{c}
\text { subgrid } \\
\text { enthalpy flux }
\end{array}}] \\
\frac{\partial \bar{\rho} \tilde{c}}{\partial t}+\frac{\partial \bar{\rho} \tilde{u}_{i} \tilde{c}}{\partial x_{i}}-\frac{\partial}{\partial x}\left(\bar{\rho} D \frac{\partial \tilde{c}}{\partial x_{i}}\right)=\underbrace{\overline{\dot{w}_{c}}}_{\begin{array}{c}
\text { filtered } \\
\text { reaction rate }
\end{array}}-\frac{\partial}{\partial x_{j}} \underbrace{\left[\bar{\rho}\left(\widetilde{u_{i} c}-\tilde{u}_{i} \tilde{c}\right)\right.}_{\begin{array}{c}
\text { subgrid } \\
\text { species flux }
\end{array}}]
\end{gathered}
$$

where $u_{i}, h_{s}, c, \lambda$, and $D$ are the $i$ th component of velocity, sensible enthalpy, reaction progress variable, thermal conductivity, and mass diffusivity, respectively. In these simulations the gas velocity did not exceed $100 \mathrm{~m} / \mathrm{s}$, which corresponds to a maximum Mach number less than 0.3 . The overline and tilde symbols refer to filtered and favre-averaged quantities, respectively. A favre-averaged variable is defined as: 


$$
\tilde{\vartheta}=\frac{\overline{\rho \vartheta}}{\bar{\rho}}
$$

The resolved viscous stress tensor, $\tilde{\sigma}_{i j}$, and the resolved strain rate, $\tilde{S}_{i j}$, are modeled as:

$$
\begin{gathered}
\tilde{\sigma}_{i j}=\mu\left(2 \tilde{S}_{i j}-\frac{2}{3} \frac{\partial \tilde{u}_{k}}{\partial x_{k}} \delta_{i j}\right) \\
\tilde{S}_{i j}=\frac{1}{2}\left(\frac{\partial \tilde{u}_{i}}{\partial x_{j}}+\frac{\partial \tilde{u}_{j}}{\partial x_{i}}\right)
\end{gathered}
$$

These governing equations are complemented by the filtered equation of state:

$$
\bar{p}=\bar{\rho} R \tilde{T}
$$

where $R$ is the gas constant based on an average molecular weight of $M W=27.74 \mathrm{~g} / \mathrm{mol}$ for both the unburned and burned gas mixtures. An average value was used to simplify the simulation and only small changes in $M W$ are expected between unburned and burned mixtures for stoichiometric methane-air combustion. The filtered reaction rate and the molecular diffusion of the reaction progress variable, $\tilde{c}$, in equation (4) are approximated using a generalized algebraic flame surface density model developed by Boger et al. [28]

$$
\frac{\partial}{\partial x}\left(\bar{\rho} D \frac{\partial \tilde{c}}{\partial x_{i}}\right)+\overline{\dot{w}_{c}}=\rho_{u} S_{L} \Sigma
$$

where $\rho_{u}=0.533 \mathrm{~kg} / \mathrm{m}^{3}$ is the density of the unburned stoichiometric methane-air mixture at an initial pressure and temperature of $p_{i}=47 \mathrm{kPa}$ and $T_{i}=293 \mathrm{~K}$, respectively. From the chemical equilibrium software, Cantera, the laminar burning velocity is estimated to be $S_{L}=47 \mathrm{~cm} / \mathrm{s}$ [29]. The flame surface density, $\Sigma$, is approximated from the following relationship:

$$
\Sigma=K_{\Sigma} \Xi \frac{\tilde{c}(1-\tilde{c})}{\Delta}
$$

where $\Xi$ is the subgrid-scale flame front wrinkling factor and $K_{\Sigma}$ is a model parameter.

Since the goal of this work is to isolate and study the effects of the unburned gas flow field development on the global flame shape, the combustion model was simplified as much as possible. As explained by Boger et al., $\Xi$ is equal to unity for a laminar, planar flame front and is expected to increase as the flame becomes wrinkled in the subgrid-scale [28]. However, it was found that $\Xi$ is relatively insensitive to turbulence levels and varies from unity to less than 1.3 over a wide range of mesh sizes. Therefore, the model is simplified in the current study by specifying a constant value of the wrinkling factor $(\Xi=1)$. Masri et al. also assumed a constant value for $\Xi$ in their LES simulation of flame acceleration around obstacles using the Boger combustion model [11]. Although a constant $\Xi$ restricts changes in the magnitude of the subgrid model's contribution to the total burning rate, it is not expected to have a large effect on the results. Instead, the interaction between the flame and the resolved scale turbulence is expected to dominate the rate of flame acceleration. This assumption is supported by the small ratio of the filter width to laminar flame thickness used in this work $\left(\delta_{L} / \Delta=0.67\right)$. Based on this resolution, the model parameter, $K_{\Sigma}$, can be estimated from the literature [28]. The direct numerical simulation (DNS) data [30,31] used to develop the Boger model resolves the flame with approximately 8 nodes. Therefore, based on the filter size used in the current study, an equivalent LES filter width to DNS mesh ratio can be determined $\left(\Delta / \Delta_{m}=5\right)$. This corresponds to $\beta=$ 0.5 and a crude estimation of the model parameter $\left(K_{\Sigma}=2\right)$. Instead of using this estimated value, $K_{\Sigma}$ was empirically fit $\left(K_{\Sigma}=0.8\right)$ to match the overall flame acceleration rate measured in one of the cases in the experimental data $(B R=0.67$ case) [4]. Note that only the overall rate of acceleration for a single obstacle $B R$ case was used in this calibration. With this calibration, the CFD model can be used to investigate the effects of obstacle BR on flame speed and further understand the development of the flame shape at the resolved scales.

Implementation of equation (10) into Fluent was facilitated through a user defined function (UDF). The heat release due to combustion, $\overline{\dot{w}_{T}}$ is calculated from: 


$$
\overline{\dot{w}_{T}}=Y_{F} \Delta H_{\text {comb }}\left(\rho_{u} S_{L} \Sigma\right)
$$

where $Y_{F}=0.05519$ and $\Delta H_{\text {comb }}=50.016 \mathrm{MJ} / \mathrm{kg}$ are the unburned mass fraction of methane and the heat of combustion for stoichiometric methane-air. The subgrid terms on the right-hand-side of equations (2)-(4) are modeled using a subgrid viscosity, $\mu_{\mathrm{SGS}}$, and a gradient diffusion approximation $[16,17]$ :

$$
\begin{gathered}
\underbrace{\bar{\rho}\left(\widetilde{u_{i} u_{j}}-\tilde{u}_{i} \tilde{u}_{j}\right)}_{\tau_{i j}}=-2 \mu_{\mathrm{SGS}} \tilde{S}_{i j} \\
\bar{\rho}\left(\widetilde{u_{i} h_{s}}-\tilde{u}_{i} \tilde{h}_{s}\right)=-\frac{\mu_{\mathrm{SGS}} C_{p}}{P r_{\mathrm{SGS}}} \frac{\partial \tilde{T}}{\partial x_{j}} \\
\bar{\rho}\left(\widetilde{u_{i} c}-\tilde{u}_{i} \tilde{c}\right)=-\frac{\mu_{\mathrm{SGS}}}{S c_{\mathrm{SGS}}} \frac{\partial \tilde{c}}{\partial x_{j}}
\end{gathered}
$$

where $P r_{\mathrm{SGS}}=0.85$ and $S c_{\mathrm{SGS}}=0.7$ are the subgrid Prandtl number and subgrid Schmidt number, respectively. In LES, the subgrid stress tensor is typically denoted as $\tau_{i j}$. The subgrid viscosity was calculated from the dynamic Smagorinsky-Lilly model $[22,23]$.

$$
\begin{gathered}
\mu_{\mathrm{SGS}}=\bar{\rho} L_{s}{ }^{2}|S| \\
L_{s}=\min \left(\kappa y_{n}, C_{s} \Delta\right) \\
|S|=\sqrt{2 \tilde{S}_{i j} \tilde{S}_{i j}}
\end{gathered}
$$

where $L_{s}, \kappa, y_{n}$ and $C_{s}$ are the subgrid turbulence length scale, von Kármán constant, normal distance to the wall, and the Smagorinsky constant, respectively. The dynamic model relies on the comparison of subgrid stresses calculated at two distinct filter widths at the same temporal and spatial position within the computational domain. The difference between the subtest-scale stress, $T_{i j}$, and the test-scale average of the subgrid stress, $\hat{\tau}_{i j}$, is defined as $L_{i j}$ :

$$
L_{i j}=T_{i j}-\hat{\tau}_{i j}=\widehat{\tilde{u}}_{i} \tilde{u}_{j}-\hat{\tilde{u}}_{i} \hat{\tilde{u}}_{j}
$$

$L_{i j}$ represents the resolved components of the stress tensor associated with scales of motion between the test scale and the grid scale and can be approximated as [22]:

$$
L_{i j}^{*}=2 C_{s}{ }^{2}\left(\hat{\Delta}^{2}\left|\hat{\tilde{S}}_{\mid} \hat{\tilde{S}}_{i j}-\Delta^{2}\right|{\widehat{\tilde{S}} \mid \tilde{S}_{i j}}\right)
$$

The following least squares method of minimizing $L_{i j}-L_{i j}^{*}$ is used to approximate $C_{s}$ :

$$
C_{s}{ }^{2}=\frac{\left\langle L_{i j} L_{i j}^{*}\right\rangle}{\left\langle L_{i j}^{*} L_{i j}^{*}\right\rangle}
$$

where \langle\rangle denotes spatial averaging in homogeneous directions.

Equations (1)-(4) were solved using the segregated pressure-based solver of the commercial CFD software, ANSYSß Fluent v.6.3. Each simulation was run in parallel on eight Intel Quad Core i-7 920 processors and took approximately one month to complete. Some of the simulations were performed on the Canadian High Performance Computing Virtual Laboratory (HPCVL). During each time-step each equation was solved sequentially and then subsequently updated using a pressure-correction equation [20]. The pressure-correction equation was implemented through the Semi-Implicit Method for Pressure-Linked Equations (SIMPLE) modified for unsteady simulations [25]. The pressure interpolation scheme and convective terms were discretized using a bounded central difference scheme, while diffusion terms were calculated using a central differencing scheme. The bounded central differencing scheme is a pure central differencing scheme that is blended with a first order upwind scheme in the event of numerical instability, which is monitored through 




Figure 3: Computational domain defined by volume outlined by dotted lines $(B R=0.5, W / H=1, L / H=$ $1.83)$.

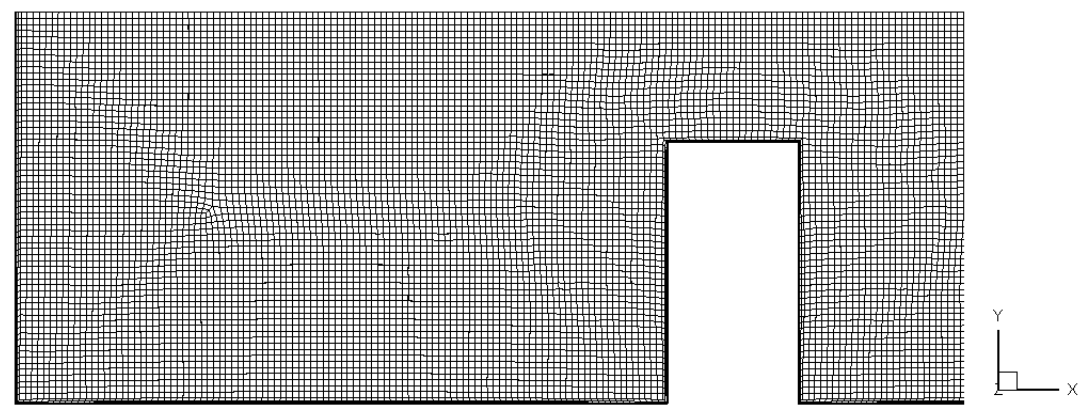

Figure 4: Computational grid at $x-y$ plane. Note that only part of the grid is shown. Dark regions near wall boundaries correspond to high aspect ratio cells $(B R=0.67, W / H=1, L / H=2.83)$.

a convection boundedness criterion [26]. Second order implicit time integration was used to discretize time derivatives.

The computational domain, outlined in figure 3 by dotted lines, corresponds to a volume spanning over two obstacle pairs downstream of the ignition point. The obstacle spacing was equal to the channel height of $H=7.62 \mathrm{~cm}$ and the length of the computational domain was varied from $L / H=2.8$ to $L / H=9.8$. The domain width was equal to the domain height $(W / H=1)$. The base grid has a node spacing of $\Delta=0.6$ $\mathrm{mm}$, which corresponds to $H / \Delta=127, \Delta / \delta_{L}=0.67$, and a total node count ranging from 1.5 million nodes $(L / H=2.8)$ to 5 million nodes $(L / H=9.8)$ in the entire domain. In these simulations the filter size is equivalent to the node spacing. Hexahedral cells with uniform node spacing in each direction were used in the domain interior and high aspect ratio cells were used near the wall surfaces. To allow for a smooth transition between the wall surface and pressure outlet at the downstream face of the computational domain, the high aspect ratio cells were extended over the pressure outlet and inflow boundary. The Courant-Friedrichs-Lewy $(C F L)$ number was monitored throughout the simulation and is defined as:

$$
C F L=\frac{U \Delta}{\Delta_{t}}
$$

where $U$ and $\Delta_{t}$ are the characteristic velocity scale and time-step size, respectively. 


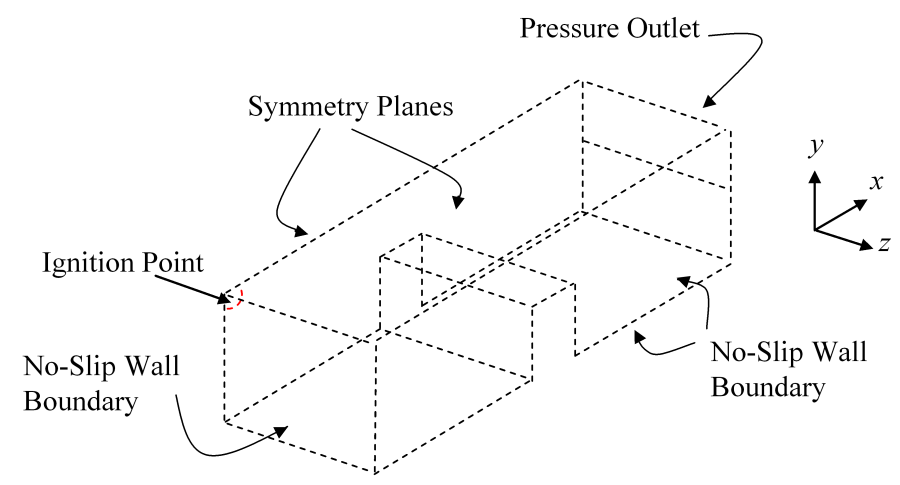

Figure 5: Boundary conditions $(B R=0.5, W / H=1, L / H=1.83)$.

As the simulation progressed, the unburned gas would accelerate and cause the $C F L$ number to increase. Therefore, the time-step was reduced periodically (approximately three instances during each simulation) to limit the maximum $C F L$ number to a value below 0.5. It was found that maintaining a maximum $C F L$ number below a value of 0.5 (e.g. $C F L<0.25)$ had a negligible effect on the flame-tip velocity development. An extensive sensitivity study of the effects of grid spacing, domain shape, and temporal resolution are available in the literature $[9,27]$.

The boundary conditions for the computational domain are shown in figure 5. Similar to the simulations of the unburned gas flow [9], the bottom surface was set to a no-slip wall boundary, which specifies zero mass flux and zero velocity [9]. The left and top boundary were specified as symmetry conditions. Based on the experimental schlieren images [4], the flame shape was observed to be symmetric as it propagated over all of the obstacles within the field of view. In addition, the flame-tip surface along the channel centerline (where the symmetry planes are located) was also observed to be laminar. Therefore, the choice to use symmetry planes instead of a full three-dimensional domain with a coarser grid resolution is justified. The right boundary was specified as a no-slip wall boundary. The pressure outlet was located above the upstream surface of the final obstacle in the computational domain and was maintained at the initial pressure and temperature of the reactants. With the use of the high aspect ratio cells near the walls, the first node off of the wall was placed within the laminar sublayer $\left(y^{+}<1\right)$. The wall shear stress, $\tau_{w}$, was obtained from the linear relationship:

$$
\tau_{w}=\mu \frac{\tilde{u}}{y}
$$

where $\tilde{u}$ is the resolved near-wall velocity. All of the subgrid terms in equations (12)-(14) are considered to be negligible in the laminar sublayer and were suppressed near the wall surfaces through equation (16). Because of the separation points at the obstacle tips, the higher grid resolution at the walls through the use of inflation layers did not have a noticeable effect on the evolution of the total flame area or the flame-tip velocity.

The pressure, temperature, velocity, and progress variable in the computational domain were initially set to $\overline{p_{i}}=47 \mathrm{kPa}, \tilde{T}_{i}=293 \mathrm{~K}, \tilde{u_{i}}=0 \mathrm{~m} / \mathrm{s}$, and $\tilde{c_{i}}=0$, respectively. The ignition source was positioned at the intersection of the end wall surface, the $x-y$ symmetry plane, and the $x-z$ symmetry plane. The initiation of the flame kernel was achieved through patching in the combustion products $(\tilde{c}=1)$ into a hemispherical volume at the ignition point. The temperature within the ignition radius was set to the adiabatic flame temperature of $T_{a d}=2205.38 \mathrm{~K}$. This value was calculated from the chemical equilibrium code, STANJAN, for a stoichiometric methane-air mixture at the simulation's initial pressure and temperature. The initial flame profile was specified using a Gaussian error function. For example, the distribution of $\tilde{c}$ at a radius, $r$, away from the ignition point is defined as: 


$$
\tilde{c}(r)=\frac{r_{i}-r}{2\left(\left|r_{i}-r\right|\right)} \operatorname{erf}\left(\frac{A_{\Delta}}{\delta_{\Delta}}\left(\left|r_{i}-r\right|\right)\right)+\frac{1}{2}
$$

where $A_{\Delta}=3.275$ is a constant. The initial patch radius was $r_{i}=6 \mathrm{~mm}$ and the initial numerical flame thickness was $\delta_{\Delta}=4 \mathrm{~mm}$. The numerical flame thickness is defined as the thickness that bounds $\tilde{c}$ from values of 0.01 to 0.99 . The initial thickness corresponds to approximately 6 cells on the base mesh $(H / \Delta$ = 127). Although more advanced spark ignition models are available in the literature [33], the model used here adequately initiates the flame and only requires a single time correction to synchronize with the experiment [4]. The initial flame kernel shape accurately represents what is observed from the experimental schlieren images shortly after ignition. Implementation of equation (23) into Fluent was facilitated through a UDF.

\section{$3 \quad$ Flame Structure Development}

Figure 6 shows the three-dimensional development of a flame propagating past four $B R=0.67$ obstacles at five different simulation times. Two images of the flame at $t=12.5 \mathrm{~ms}$ are displayed to assist the interpretation of the images at later times in the figure. The left image shows the distribution of an isosurface of the reaction progress variable $(\tilde{c}=0.5)$ created by reflecting the computational domain over each of the symmetry planes shown in figure 5 . The distribution of the $\tilde{c}=0.5$ iso-surface, which is representative of the flame surface, is superimposed onto a light grey wire frame, representing the computational domain. The flame-tip at this simulation time had not yet reached the first obstacle pair. The right image at $t=12.5$ ms shows $x-y$ and $y-z$ planes positioned along the symmetry planes of the computational domain so that the internal flame structure can be observed. Contours of $\tilde{c}$ are shown on these planes and stream-traces are overlaid to show the instantaneous paths of a particle through the flow field at each given time.

At $t=15 \mathrm{~ms}$, the flame-tip accelerates through the opening between the obstacles, which stretches the flame and increases the flame area. This phenomenon is discussed in more detail in the literature [4]. The divergence of stream-traces is observed along the lateral flame surface near the channel's top and bottom surfaces at the ignition end of the channel. On the burned side of the flame, the expansion of hot gas drives the flow inwards, away from the flame surface (i.e., stream-traces point towards the center of the channel and then turn towards the end of the channel). On the unburned side, the flame acts as a piston, driving the unburned gas outwards and away from the flame (i.e., initially stream-traces point towards the channel walls and then turn towards the end of the channel). Similar to the predictions from the numerical simulations of the unburned gas flow by Johansen and Ciccarelli [9], laminar recirculation zones grow in the unburned gas after each obstacle. Once the flame reaches the recirculation zone after the first obstacle pair, it entrains into the vortex, as seen in the roll-up of the flame surface at $t=17.5 \mathrm{~ms}$.

Up to a simulation time of $t=17.5 \mathrm{~ms}$, the flame on the $x-z$ plane is parabolic in shape due to the wall friction on the side walls. A small kink forms in the lateral flame surface between the first and second obstacles $(t=20 \mathrm{~ms})$ and grows $(t=21.25 \mathrm{~ms})$, causing lateral deformation of the stream-traces. These deformations in flame shape occur due to the three-dimensional effects of accelerating flow through the obstacle gaps. As the flame-tip approaches the opening between obstacles, the centerline becomes stretched, narrowing the diameter of the flame cross-section. This deformation occurs both in the $x-y$ and $x-z$ planes. As the flame rolls up into the vortex downstream of the obstacle, it is able to propagate laterally, closer to the channel side walls. This type of development in the flame shape changes at later times when compressibility effects become dominant [5].

Near the end of the simulations $(t=21.25 \mathrm{~ms})$, the flame shape becomes stretched in the $x$ direction and does not immediately propagate into recirculation zones between the obstacles. Thin filaments in the flame surface, however, appear to jet into the recirculation zone resulting in highly contorted structures, which occur at small length scales. The centerline flame velocity near the end of the computational domain in Fig. 6 reaches approximately $80 \mathrm{~m} / \mathrm{s}$. Furthermore, small isolated pockets of burned gas begin to appear in the unburned gas outside of the main flame front. The breakup of the flame surface at later times is possibly an indication that a transition between turbulent combustion regimes is beginning to occur. Since the flame surface density models are only intended to operate within the "thin reaction zone regime", a transition to a "broken reaction zone regime" would render the assumptions of the model invalid. In addition, since a 




Figure 6: 3D evolution of $\tilde{c}=0.5$ iso-surface $(B R=0.5, W / H=1, L / H=5.83)$. Contours of $\tilde{c}$ shown on $x-y$ and $x-z$ planes positioned at the symmetry planes of the computational domain. Stream-traces overlaid onto $x-y$ and $x-z$ planes. (This image should be published in color) 
low-Mach number formulation of the governing equations was solved, compressibility effects in the energy equation were not accounted for. Therefore, this modelling approach appears to be limited to flame speeds below $80 \mathrm{~m} / \mathrm{s}$.

A global indicator of the type of turbulent combustion regime that exists is the Karlovitz number, $K a$, which is defined as:

$$
K a=\frac{\tau_{c}}{\tau_{\eta}}=\frac{\left(\delta_{L} / S_{L}\right)}{\left(\eta / u_{\eta}^{\prime}\right)}
$$

where $\tau_{\mathrm{c}}, \tau_{\eta}, \delta_{L}, \eta$, and $u_{\eta}^{\prime}$ are the chemical time scale, Kolmogorov time scale, laminar flame thickness, Kolmogorov length scale, and Kolmogorov velocity scale, respectively. Pitsch and Duchamp de Lageneste [32] show that for a filter width selected within the inertial subrange of the turbulence energy spectrum, $K a$ can be estimated from subgrid quantities:

$$
K a=\left(\frac{v^{\prime} \Delta}{S_{L}}\right)^{\frac{3}{2}}\left(\frac{\delta_{L}}{\Delta}\right)^{\frac{1}{2}}
$$

where $v^{\prime}{ }_{\Delta}$ is the subgrid velocity scale. Pitsch and Duchamp de Lageneste comment that although equation (25) is a function of filter width, $v^{\prime}{ }_{\Delta}$ naturally compensates for changes in $\Delta$ so that a constant value of $K a$ is maintained. This assumption, of course, is dependent on the quality of the subgrid model. Based on the characteristic time and length scales of the dynamic Smagorinsky-Lilly subgrid model [17], $\mathrm{Ka}$ can be reformulated as follows:

$$
K a=\left(\frac{C_{s} \Delta|S|}{S_{L}}\right)^{\frac{3}{2}}\left(\frac{\delta_{L}}{\Delta}\right)^{\frac{1}{2}}
$$

Figure 7 shows the spatial distribution of $K a$ as a contour plot on an iso-surface of the progress variable for a simulation time of $t=22.5 \mathrm{~ms}$. The absence of turbulence near the ignition end of the channel results in a smooth flame surface and corresponding low levels of $K a$ (blue contour). The flame surface near the third obstacle has higher levels of $K a$ due to high levels of strain-rate and subgrid viscosity predicted in the shear layer. Karlovitz numbers within this region are limited to approximately $K a<90$. Near the flame-tip, high levels of $K a$ (red contour) form in localized patches along the flame surface in the shear layer. The spatial distribution is highly unsteady and the maximum levels increase as the simulation progresses. Figure 8 shows the type of turbulent combustion regime that is being modeled as the simulation progresses in time. Red points located on the regime diagram are based on maximum levels of Ka calculated from equation (26). These points are typically located near the flame-tip along the shear layer. Since the filter width was held constant throughout the simulation, the value of $\Delta / L=0.67$ did not change as levels of turbulence increased.

At $t=22.5 \mathrm{~ms}$, the maximum Karlovitz number was $K a=288$, which placed a localized region of the flame within the "broken reaction zone regime". Therefore, as the simulation progresses in time, one would expect a transition to occur from a "laminar flamelet regime", to a "thin reaction zone regime", and finally to a "broken reaction zone regime". For simulation times up to $t=21.25 \mathrm{~ms}$, however, the entire flame surface is within the "laminar flamelet" or "thin reaction zone regimes".

\section{Simulation Comparison to Experimental Results}

The comparisons between the experiment and the simulation predictions are based on flow visualization images. Figure 9 shows the predictions of the flame shape in the $x-y$ plane obtained from simulations for several obstacle $B R$ configurations. Simulations are compared to the schlieren images obtained from the experiment and have a corresponding grid resolution of $H / \Delta=127$. For each experimental image shown, the corresponding simulation image was selected to match the flame-tip position. In general, the simulation times, $t$, were observed to be approximately $3.5 \mathrm{~ms}$ behind that of the experiments. At $t=0 \mathrm{~ms}$, the simulated flame-tip position is not at $x / H=0$ due to the flame surface initialization method. In addition, in the experiment there is significant heat transfer between the flame surface and spark plug shortly after ignition. The simulation does not include the flame development at the spark plug nor does it include 


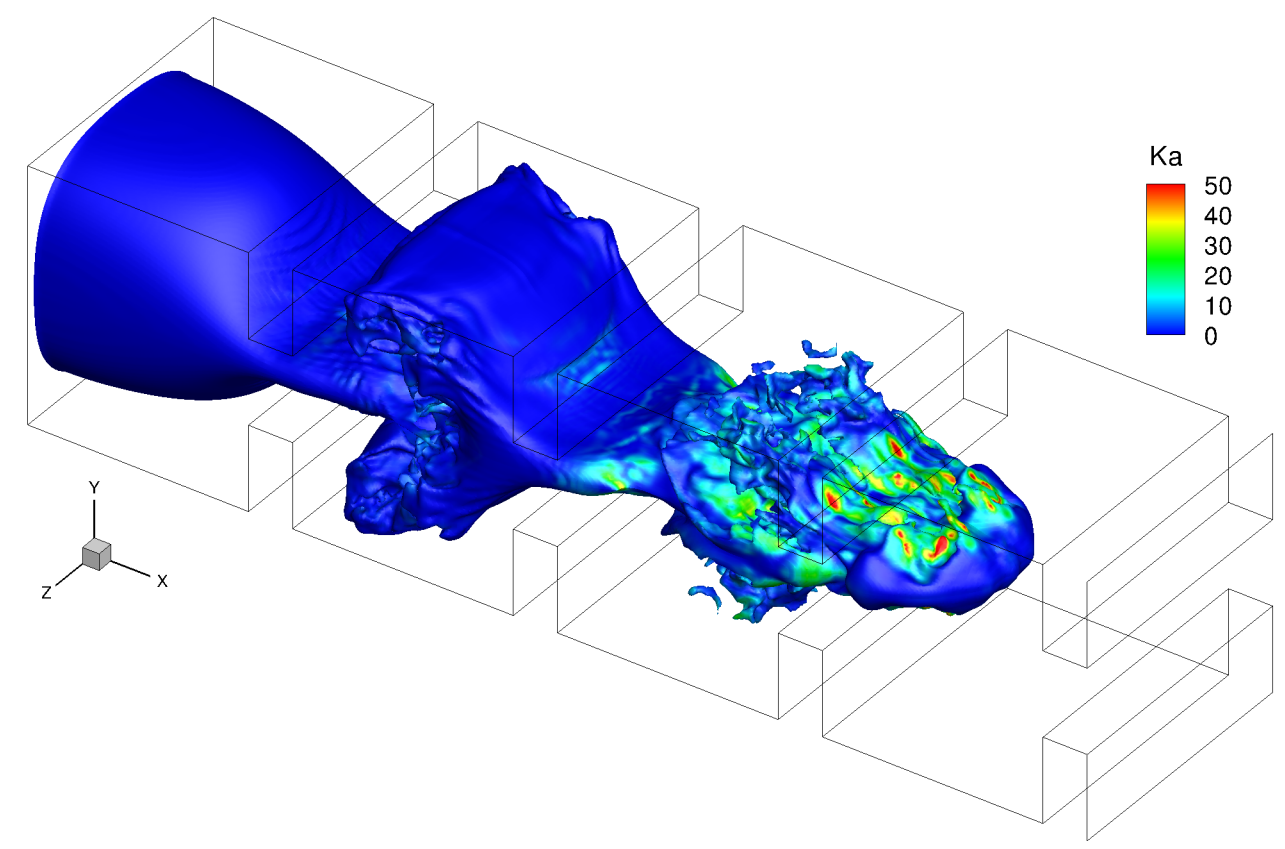

Figure 7: Spatial distribution of the Karlovitz number over the flame surface $(\tilde{c}=0.5)$ calculated from equation $(26)(B R=0.67, W / H=1, L / H=5.83, t=21.25 \mathrm{~ms})$ (This image should be published in color)

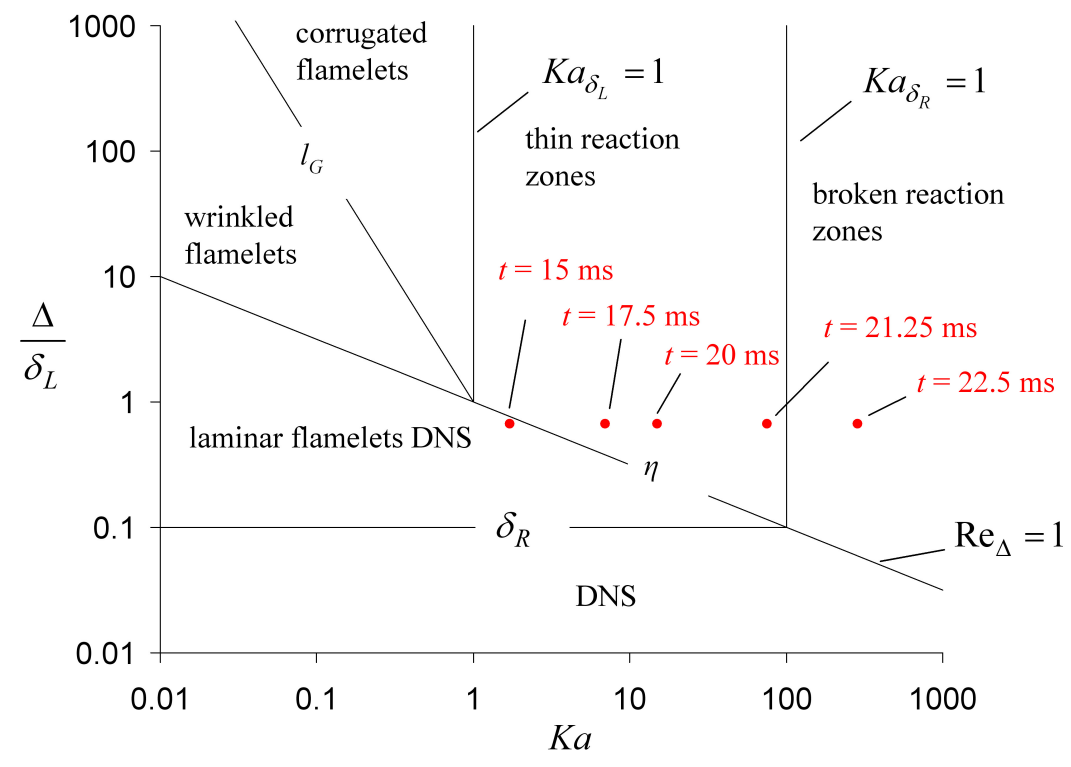

Figure 8: Range of turbulence combustion regimes occurring in the unsteady simulation $(B R=0.67, W / H$ $=1, L / H=5.83)$. Red points indicate the maximum Karlovitz numbers calculated at different simulation times, which correspond to the images in Fig. 6. Limits of the combustion regimes are reproduced from Ref. [32]. 

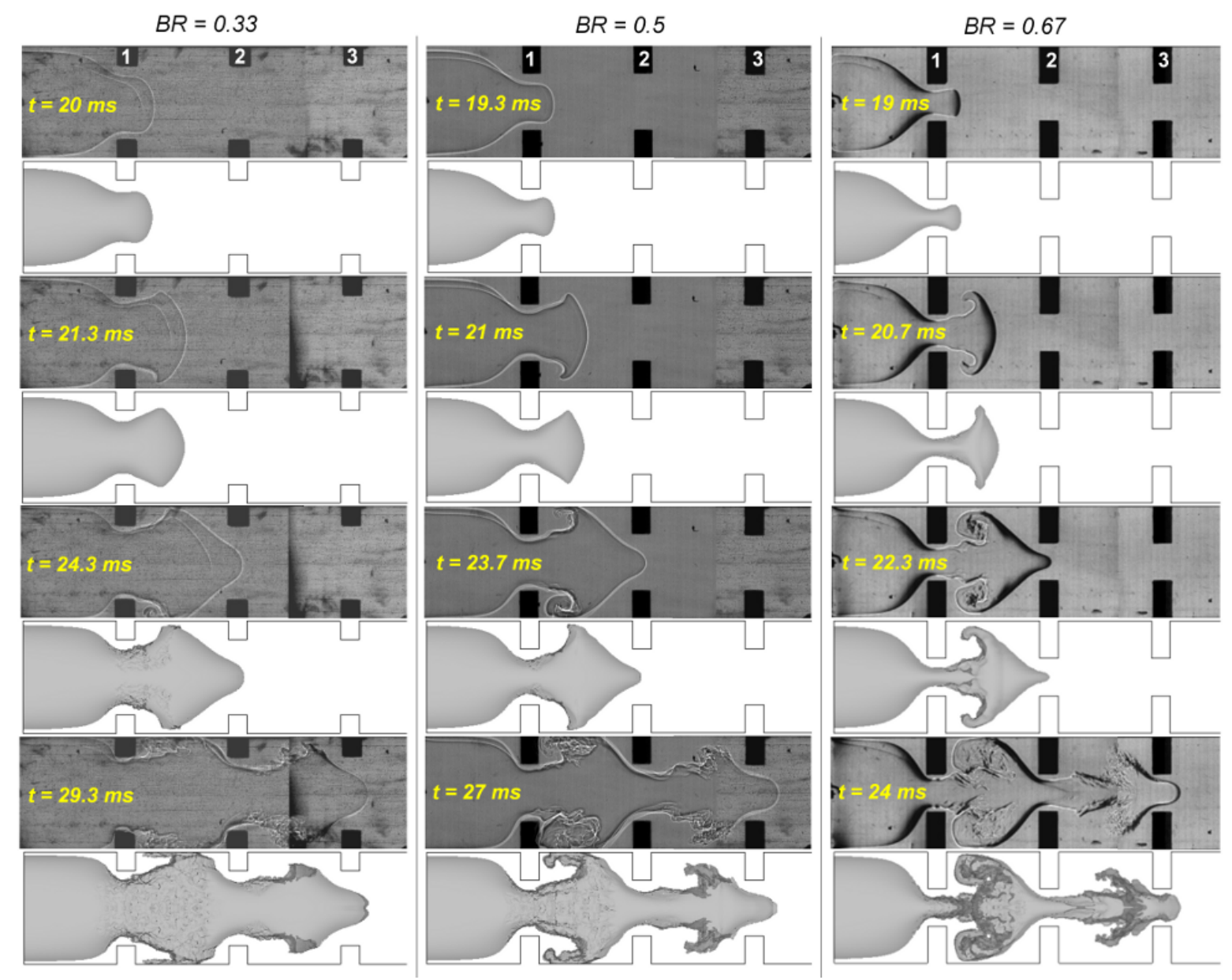

Figure 9: Simulation predictions of flame shape $(\tilde{c}=0.5$ iso-surface $)$ compared to schlieren images. Time indicated on schlieren images. BOGER Flame Surface Density Model is used $(B R=0.67, W / H=1, L / H$ $=9.83)$. Obstacle numbers shown in white.

heat transfer to the wall boundaries. After an initial modification to the simulation time (add $3.5 \mathrm{~ms}$ ), the experiment and simulation remain roughly synchronized over several obstacle pairs for each obstacle $B R$ configuration. Since the schlieren images were created by integrating all of the density gradients over the entire channel width $(W / H=1)$, the simulation results are presented as a projected three-dimensional iso-surface $(\tilde{c}=0.5)$ onto the $x-y$ plane. The maximum density gradients in the simulation coincide with progress variable values near $\tilde{c}=0.5$. The iso-surfaces are colored in a grey scale to mimic the black and white schlieren photos and were translucent so that the internal structure was visible beneath the outer three-dimensional flame surface.

In general there is good agreement between the predicted flame shape and the experiments. There is some asymmetry in the experimental images, which is amplified in cases with lower $B R$ obstacles. This is caused by the non-symmetrical shape of the spark plug and buoyancy forces that are significant during the initial growth of the combustion kernel. As soon as the flame propagates past the first obstacle, the overall symmetry of the flame shape returns. As indicated by the reported times in the figure, the higher contraction ratio results in a higher flame-tip velocity. Both the experiments and simulations show that at high obstacle $B R$, the shear layer that forms from the obstacle tips is able to penetrate further into the core. The formation of turbulence in these regions causes small scale three dimensional contortions of the flame surface. As a result, it is expected that a two dimensional simulation would be unable to accurately capture the growth in flame area and subsequent flame-tip acceleration rate. Further analysis of this issue is discussed in more 


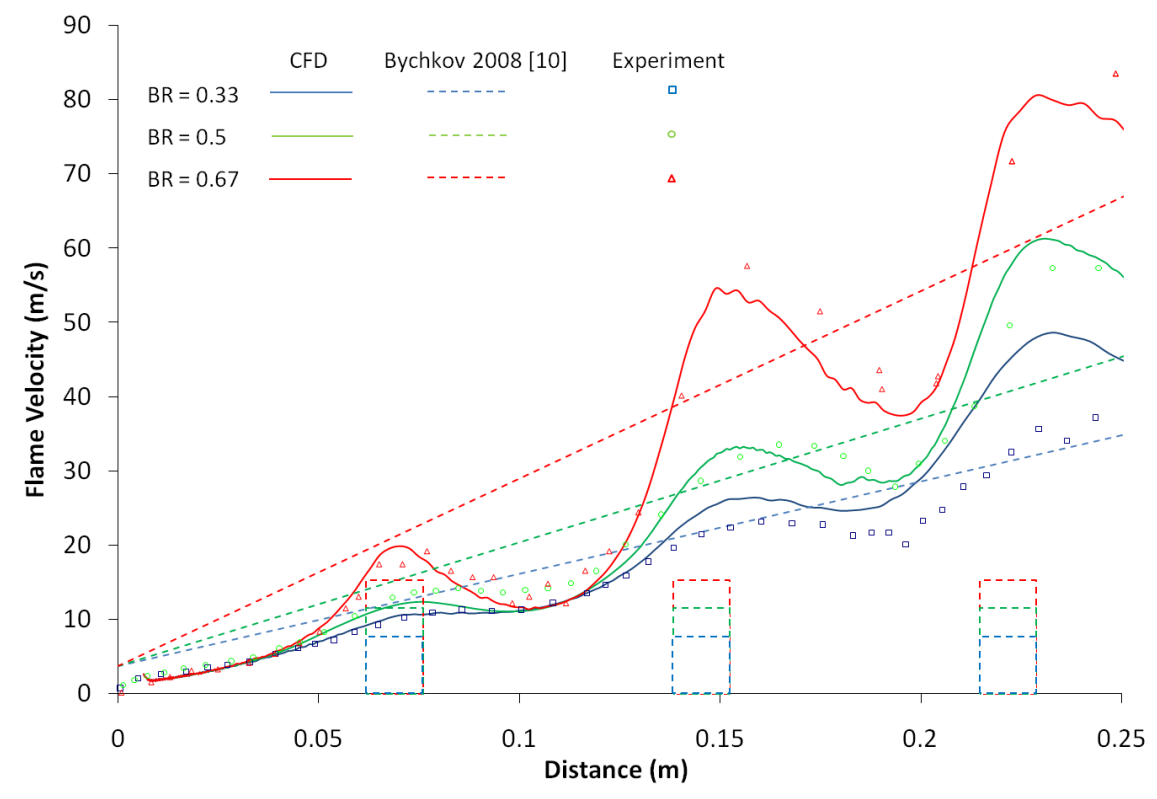

Figure 10: Simulation predictions of the centerline flame velocity. BOGER Flame Surface Density Model used $(W / H=1, L / H=5.83)$.

detail in the literature [27]. Many of the unique flame structures observed experimentally are reproduced in the simulations. As expected, this observation indicates that the development of the unburned gas flow field ahead of the flame plays a large role in determining the overall flame shape. Note that in each $B R$ case the flame tip remains laminar at all times reported. There is, however, a small inflection in the flame-tip near the centerline for the $B R=0.33$ case $(t=27 \mathrm{~ms})$. This inflection only occurs in cases with low $B R$ obstacles. It appears in cases with more refined grids $(B R=0.33)$ and in cases with varying domain lengths. The root cause of this phenomenon is unclear and will be investigated in future work. As a result, quantitative measurements including flame velocity and flame area will not be reported for flame-tip positions further downstream than the third obstacle.

Figure 10 shows the simulation predictions of the increase in the centerline flame-tip velocity corresponding to each obstacle $B R$ configuration. There is good agreement between the simulation predictions and experiment. Included in the figure is an analytical expression for the flame-tip velocity developed by Bychkov (2008) [8]:

$$
U_{f}=\frac{d Z_{f}}{d t}=\frac{2(\sigma-1) S_{L}}{(1-B R) H} Z_{f}+\sigma S_{L}
$$

where $U_{f}, Z_{f}$, and $\sigma$ are the flame-tip speed, flame-tip position, and expansion ratio, respectively. The expansion ratio $(\sigma=7.56)$ was determined from the chemical equilibrium software STANJAN. The model assumes that the initial rate of flame acceleration is dominated by the powerful jets created from the delayed burning of reactants between obstacles behind the flame front. In addition, it assumes that wall friction and turbulence effects play only a secondary role in affecting the flame-tip speed. Because of the simplicity of the model, the pulsation of the flame-tip velocity due to flow contraction and expansion was not captured.

Figure 11 shows predictions of the flame area growth for each obstacle $B R$ configuration. Experimentally, only one configuration $(B R=0.67)$ was analyzed to extract the flame area. To extract the flame area from the schlieren images, both $x-y$ and $x-z$ views of the flame are required. This was only performed for the $B R=0.67$ configuration. In the simulations the iso-surface area corresponding to the center of the flame $(\tilde{c}$ $=0.5)$ was reported. This does not include the contribution of the any subgrid-scale wrinkling to the total flame area. The simulation results show a slight under-prediction of the flame area, which is confirmed by observing the flame shape prediction in figure 9 . At $t=24 \mathrm{~ms}(B R=0.67)$, for example, the cross-section of 


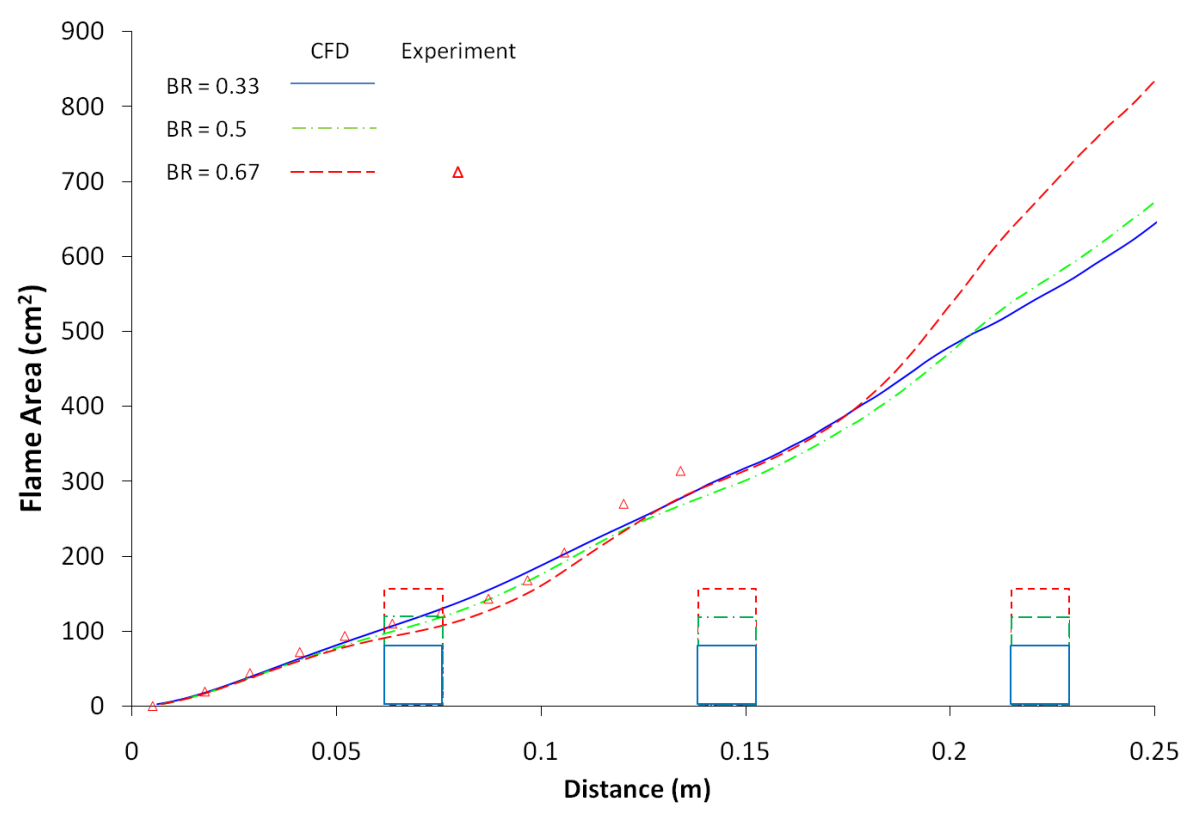

Figure 11: Simulation predictions of the flame area. BOGER Flame Surface Density Model used $(W / H=$ $1, L / H=5.83)$.

the flame-tip downstream of obstacle \#2 in the $x-y$ plane was smaller than the cross-section of the flame-tip in the corresponding schlieren image. In addition, the lateral flame surface was much closer to the channel top and bottom walls in the schlieren images compared to the simulations. It appears that the simulations over-predict the stretch rate of the flame structure in the x-direction, which results in an under-prediction of the flame area for a given flame-tip position.

\section{$5 \quad$ Analysis of Simulated Flow Field}

Based on good agreement between the simulation and experiment with regards to the flame shape, oscillations in flame-tip velocity, and growth of the flame area, the solutions of the simulation can be analyzed further to understand more about the physics of the problem. The roll-up of the vortex downstream of obstacle \#1 (Fig. 9), for example, is a feature predicted in the simulations. The extent of the entrainment of the flame surface into the vortex roll-up, however, was smaller in the simulations compared to the schlieren images. Downstream of obstacle \#2, the development of the shear layer resulted in a different flame shape than what is observed downstream of obstacle \#1. Figure 12 shows the formation of a "mushroom"-shaped feature just before the end of the third obstacle. The mushrooming shape is explained by investigating the interaction of the flame with the developing recirculation zone.

Figure 13 shows a sequence of predicted vorticity distributions in both the unburned gas and combustion products as the flame-tip approaches the outflow boundary through the third obstacle. The inter-frame time of these images is $250 \mu \mathrm{sec}$. Stream-traces and the projection of the translucent $\tilde{c}=0.5$ iso-surface show the velocity flow field and flame structure, respectively. The "mushrooming" effect observed in the schlieren images is due to the convection of the flame-tip along the shear layer, which follows the shape of the large recirculation zone. In each of the images shown in Fig. 13, the expansion of the stream-traces occurs near the downstream side of the recirculation zone, which is caused by the rotational velocity of the vortex. Near the upstream side of the recirculation zone, stream-traces are roughly parallel in the horizontal direction. Therefore, the flame-tip appears elongated as it passes through obstacle \#2 $(t=23.2 \mathrm{~ms})$ because it has not reached the downstream side of the recirculation zone, where expansion occurs. The expansion of the flame surface downstream of the second obstacle becomes noticeable roughly at $t=23.45 \mathrm{~ms}$ in Fig. 13 . At this time, the distribution of $\tilde{c}$ along the lateral flame surface near the flame-tip becomes highly wrinkled in each 


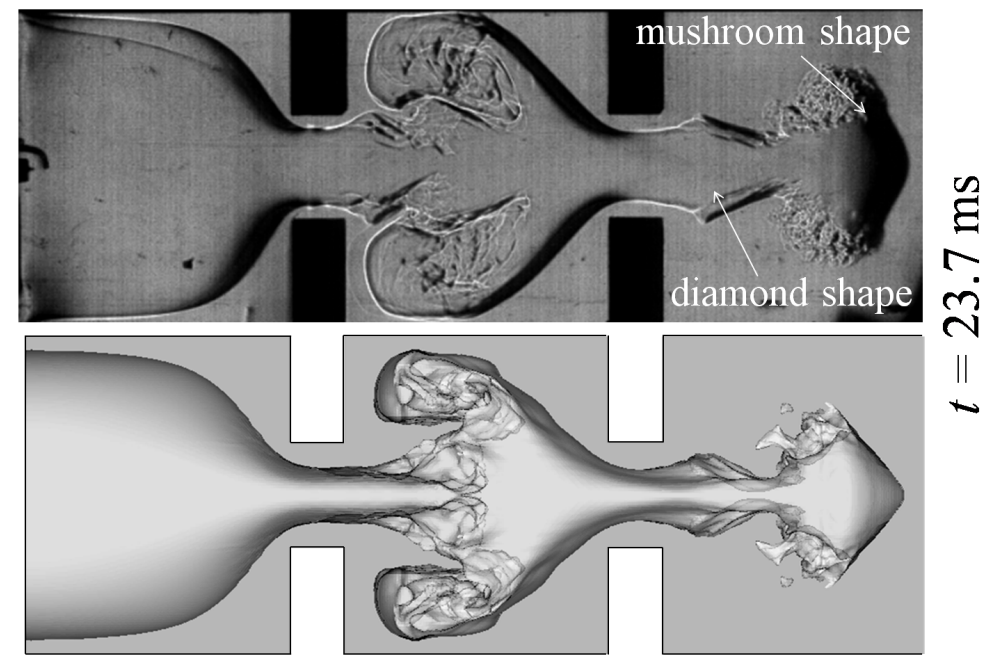

Figure 12: Mushroom effect $(B R=0.67, W / H=1, L / H=2.83, t=23.7 \mathrm{~ms})$.

of the three spatial dimensions. These three-dimensional distortions in the flame surface coincided with the breakdown of larger, mainly two-dimensional vortices, into smaller three-dimensional vortices. Note that the unburned gas velocity magnitude is lower in the recirculation zones compared to the core flow. As a result, the lateral flame surface is more susceptible to distortions from lower strength vortices in the recirculation zone. Note that the flame tip (top of the mushroom) remains perfectly laminar as observed in the schlieren image in Fig. 12. The shape of the tip is governed by the expansion and then the contraction of the stream lines at each obstacle.

A diamond shaped feature, which is just upstream of the mushroom shape (Fig. 12), is observed in the experiment and predicted in the simulations. This phenomenon is also due to the development of the recirculation zones. At $t=23.2 \mathrm{~ms}$ (Fig. 13), the flame surface slowly expands near the flame-tip due to the formation of the main recirculation zone. Only small deformations existed along the lateral edge of the flame surface at this time. Later, at $t=23.7 \mathrm{~ms}$, the center of the main vortex associated with the recirculation zone convects downstream and a small secondary vortex forms at the trailing edge of obstacle \#2. This augments the stream-traces toward the channel surfaces and the flame shape propagates accordingly in the transverse direction. The stream-traces then redirect back towards the channel centerline as the velocity associated with the upstream side of the main recirculation zone points towards the channel centerline. As a result, a diamond shape appears in the flame surface downstream of the second obstacle at $t=23.7 \mathrm{~ms}$.

Figure 14 shows the evolution of the centerline gas velocity along the length of the channel $(B R=0.67)$ at two simulation times. At $t=23.2 \mathrm{~ms}$, an oscillatory motion in the centerline gas velocity is observed ahead of the flame. At the ignition end of the channel, the gas velocity is close to stationary due to the presence of the wall boundary. Since combustion still occurs at the lateral flame surface at the ignition end, a positive centerline gas velocity is observed between the ignition point and the flame-tip. In addition, since the burned gas flow also experiences flow contraction and expansion near obstacles, an oscillatory pattern is also observed in the burned gas velocity. The peak velocity in the burned gas occurs at the second obstacle and exceeds each of the velocity peaks in the unburned gas. A higher burned gas velocity is due to the acceleration of the lower density combustion products behind the flame-tip, which is driven by combustion in the recirculation zones behind obstacle \#2. As the flame-tip accelerates, the regular oscillating motion of the unburned gas velocity degenerates due to instabilities and vortex shedding in the shear layer, which is also observed in simulations of the unburned gas flow [9]. At $t=23.95 \mathrm{~ms}$ in Fig. 14, the oscillations in the unburned gas velocity do not appear regular and do not appear to amplify. Experimental data, however, shows large oscillations in the flame-tip velocity at obstacles further downstream [5]. The mechanism for these oscillations is mainly related to the interaction of the flame with compression waves that reflect back from upstream obstacles. As discussed above, that mechanism is not modeled in the present simulations. 

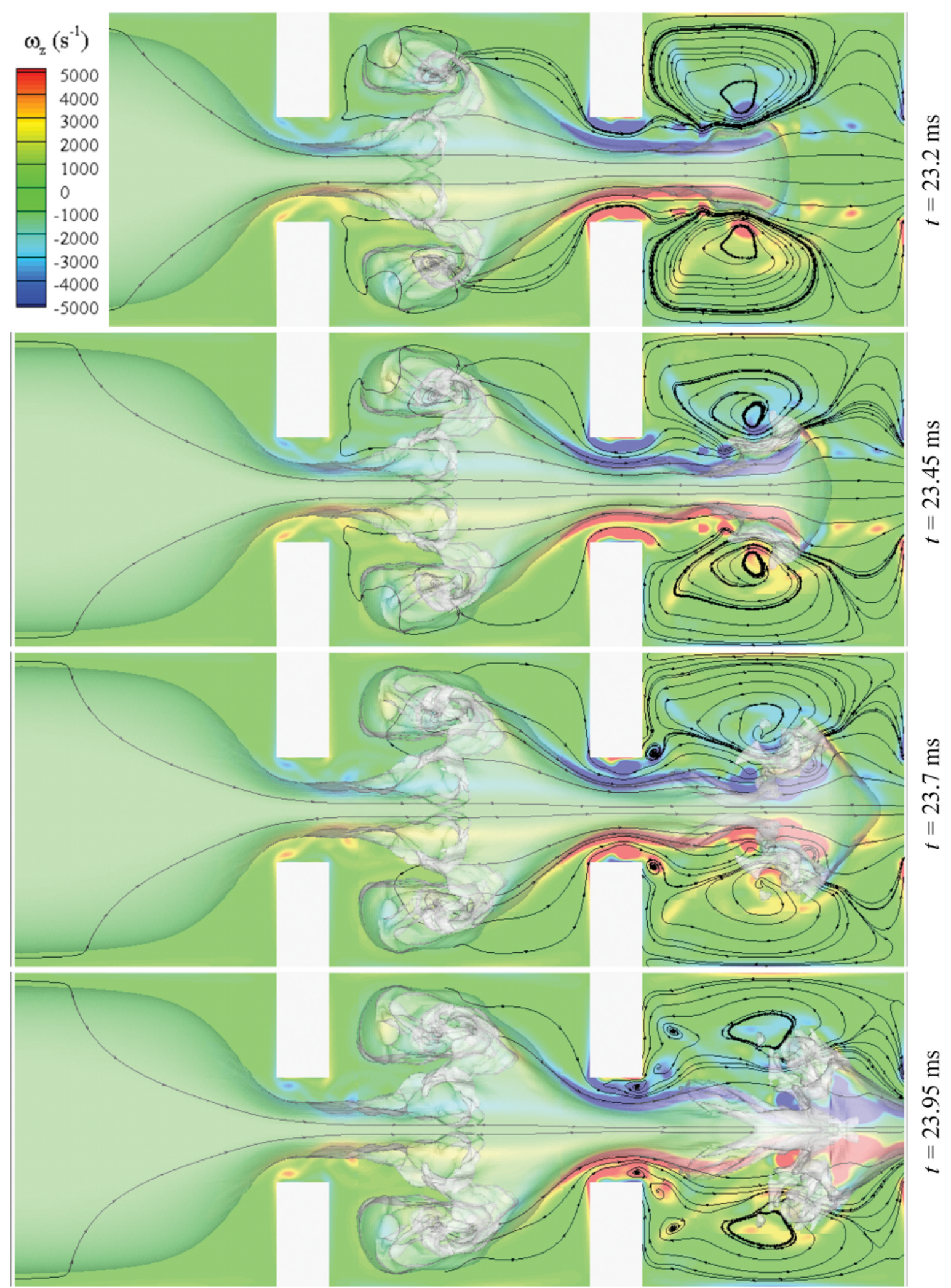

Figure 13: Distribution of vorticity for flame development after second obstacle $(B R=0.67, W / H=1, L / H$ $=2.83$ ). Stream-traces and $\tilde{c}=0.5$ iso-surface overlaid onto the vorticity contour. BOGER Flame Surface Density Model is used. (This image should be published in color) 

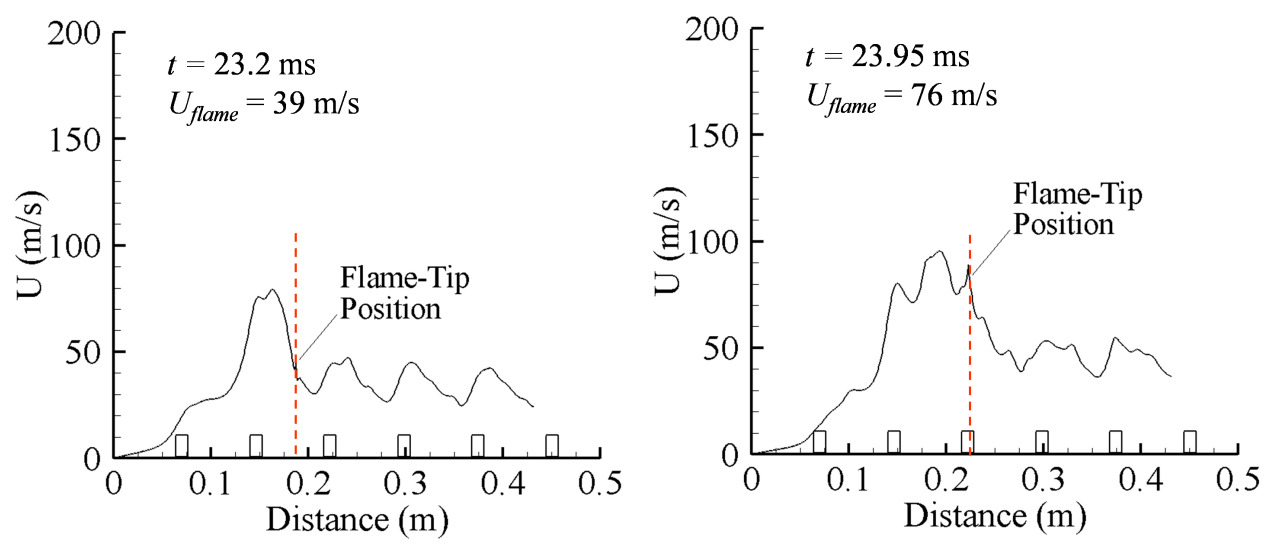

Figure 14: Simulation predictions of centerline gas velocity $(B R=0.67, W / H=1, L / H=5.83)$. Flame-tip position shown as dashed line.

\section{Conclusions}

Three-dimensional large eddy simulations of flame propagation in a closed, obstructed channel with various obstacle blockage ratios were performed using a relatively simple flame surface density combustion model. The flame is able to accelerate up to two orders of magnitude above the laminar burning velocity by convecting on the unburned gas flow. During this acceleration period, it was determined that combustion remains within the "laminar flamelet" and "thin reaction zone" regimes. Based on good agreement between experiments and simulation predictions, it was found that the development of the flame shape, acceleration of the flame-tip, and growth of the flame area during the initial stages of flame acceleration are mainly influenced by the resolved unburned gas flow field rather than by the subgrid combustion model. Simulation results indicate that the flame shape becomes stretched in the stream-wise direction due to interactions with small vortical structures that form in the shear layer. The development of these three-dimensional flow structures in the unburned gas inhibit burning near the wall surfaces and result in a highly wrinkled transverse (or lateral) flame surface. The simulations show that an increase in flame area and subsequent acceleration of the unburned gas flow through high contraction-ratio openings causes faster rates of flame acceleration to occur in the higher $B R$ obstacle configurations. It was found that the flame interaction with small recirculation zones, immediately downstream from the obstacles, at low flame speeds results in a relatively smooth rollup of the flame surface. At higher flame speeds, a "mushroom" shape of the flame-tip is observed both experimentally and numerically and occurs due to flame interaction with a much larger recirculation zone. Overall, the simulation results demonstrate that the majority of features of the flame shape observed during the initial stages of flame acceleration form from the interaction of the flame with an evolving recirculation zone structure between obstacles.

\section{Acknowledgements}

This work was supported by the Natural Sciences and Engineering Research Council of Canada. The authors would like to thank Dr. Mike Birk for the use of his computing cluster for some of the simulations. Simulations were also run using the Canadian HPCVL computing resources.

\section{References}

[1] G. Ciccarelli and S. Dorofeev, Flame acceleration and transition to detonation in ducts, Prog. Energy Combust. Sci. 34 (2008), pp. 499-550. 
[2] G.D. Roy, S.M. Frolov, A.A. Borisov, and D.W. Netzer, Pulsed detonation propulsion: challenges, current status, and future perspective, Prog. Energy Combust. Sci. 30 (2004), pp. 545-672.

[3] K.I. Shchelkin, Influence of tube roughness on detonation formation and propagation in gases, J. Exp. Theor. Phys. 10 (1940), pp. 823-827.

[4] C. Johansen and G. Ciccarelli, Visualization of the unburned gas flow field ahead of an accelerating flame in an obstructed square channel, Combust. and Flame 156 (2009), pp. 405-416

[5] G. Ciccarelli, C. Johansen, and M. Parravani, The role of shock-flame interactions on flame acceleration in an obstacle laden channel, Combust. and Flame 157 (2010), pp. 2125-2136.

[6] S.M. Frolov, Liquid-fueled, air-breathing pulse detonation engine demonstrator: operation principles and performance, J. Propuls. Power 22 (2006), pp. 1162-1169.

[7] V. Gamezo, O. Takanobu, and E.S. Oran, Numerical simulations of flame propagation and DDT in obstructed channels filled with hydrogen-air mixture, Proc. Combust. Inst. 31 (2007), pp. 2463-2471.

[8] V. Bychkov, D. Valiev, and L. Eriksson, Physical mechanism of ultrafast flame acceleration, Phys. Rev. Lett. 101 (2008), pp. 1-4.

[9] C. Johansen and G. Ciccarelli, Numerical simulations of the flow field of an accelerating flame in an obstructed channel, Combust. Theor. Model. 14 (2010), pp. 235-255.

[10] V. Di Sarli, A. Di Benedetto, G. Russo, S. Jarvis, E.J. Long, and G.K. Hargrave, Large eddy simulation and PIV measurements of unsteady premixed flames accelerated by obstacles, Flow, Turb. and Combust., DOI 10.1007/s10494-008-9198-3 (2009), pp. 1-24.

[11] A.R. Masri, S.S. Ibrahim, and B.J. Cadwallader, Measurements and large eddy simulation of propagating premixed flames, Exp. Therm. Fluid Sci. 30 (2006), pp. 687-702.

[12] S.S. Ibrahim, S.R. Gubba, A. Masri, and W. Malalasekera Calculations of explosion deflagrating flames using a dynamic flame surface density model, Loss. Prevention. 22(3) (2009), pp. 258-264.

[13] S.R. Gubba, S.S. Ibrahim, W. Malalasekera, and A.R. Masri, Measurements and LES calculations of turbulent premixed flame propagation past repeated obstacles, Combust. and Flame 158(12) (2011), pp. $2465-2481$.

[14] X. Wen, M. Yu, Z. Liu, and W. Sun, Large eddy simulation of methane-air deflagration in an obstructed chamber using different combustion models, Loss. Prevention. 25, (2012), pp. 730-738.

[15] S.N.D.H., Patel, S. Jarvis, S.S. Ibrahim, G.K. Hargrave, An experimental and numerical investigation of premixed flame deflagration in a semiconfined explosion chamber, Proc. Combust. Inst. 29 (2002), pp. 1849-1854.

[16] T. Poinsot and D. Veynante, Theoretical and Numerical Combustion, RT Edwards, Flourtown, PA, 2001.

[17] E. Garnier, N. Adams, and P. Sagaut, Large Eddy Simulation for Compressible Flows, Springer-Verlag, New York, 2009.

[18] J. Smagorinksy, General circulation experiments with the primitive equations. I. The basic experiment., Month. Weath. Rev. 91 (1963), pp. 99-164.

[19] S.B. Pope, Turbulent Flows, Cambridge University Press, New York, 2000.

[20] Fluent Inc. Fluent User Manual, Lebanon, NH: Fluent Inc., (2007).

[21] J.H. Ferziger, Subgrid-scale modeling, in Large Eddy Simulation of Complex Engineering and Geophysical Flows, B. Galperin and S.A. Orszag, Cambridge University Press, Cambridge, 1993, pp. 37-54. 
[22] M. Germano, U. Piomelli, P. Moin, and W. H. Cabot, A dynamic subgrid-scale eddy-viscosity model, Phys. Fluids A 3 (1991), pp. 1760-1765.

[23] D.K. Lilly, A proposed modification of the Germano subgrid-scale closure model, Phys. Fluids A 4 (1992), pp. $633-635$.

[24] L. Marstorp, Subgrid-scale modelling for large eddy simulation including scalar mixing in rotating turbulent shear flows, Technical Reports from the Royal Institute of Technology, KTH Mecahnics (2006), pp. 1-64.

[25] S.V. Patankar and D.B. Spalding, A calculation procedure for heat, mass and momentum transfer in three-dimensional parabolic flows, Int. J. Heat Mass Transfer 15 (1972), pp. 1787-1806.

[26] B.P. Leonard, The ultimate conservative difference scheme applied to unsteady one-dimensional advection, Comp. Math. Appl. Mech. Eng. 88 (1991), pp. 17-74.

[27] C.J. Johansen, Experimental and numerical investigation of flame acceleration in an obstructed channel, PhD Thesis Dissertation, Mech. and Materials Eng., Queen's University, 2009.

[28] M. Boger, D. Veynante, H. Boughanem, A. Trouve, Direct numerical simulation analysis of flame surface density concept for large eddy simulation of turbulent premixed combustion, 27th Symp. Comb., Colorado, 1998.

[29] D.G. Goodwin, Cantera: Object-oriented software for reactive flows Retrieved Sept 1, 2009, from http://www.cantera.org.

[30] H. Boughanem, Evaluation des Termes de Transport et de Dissipation de Surface de Flamme par Simulation Numerique de la Combustion Turbulente, PhD Thesis Dissertation, University of Rouen, 2009.

[31] H. Boughanem and A. Trouve The domain of influence of flame instabilities in turbulent premixed combustion, 27th Symp. Comb., Colorado, 1998.

[32] H. Pitsch and L. Duchamp de Lageneste, Large eddy simulation of premixed turbulent combustion using a level-set approach, Proc. Combust. Inst. 29 (2002), pp. 2001-2008.

[33] O. Colin and K. Truffin, A spark ignition model for large eddy simulation based on an FSD transport equation (ISSIM-LES), Proc. Combust. Inst. 33 (2011), pp. 3097-3104. 